\title{
Indicação de punção lombar para diagnóstico da neurossífilis
}

\author{
Indication of lumbar puncture for neurosyphilis diagnosis
}

Filipe Augusto Gava Martins ${ }^{1}$, Bernardino Geraldo Alves Souto ${ }^{1}$

\section{RESUMO}

A sífilis é um agravo infectocontagioso causado pelo Treponema pallidum, de transmissão predominantemente sexual, que afeta 12 milhões de pessoas por ano no mundo. Se não curada em suas fases iniciais, predispõe a complicações potencialmente graves. Uma dessas complicações é a neurossífilis, caracterizada pela invasão do sistema nervoso central pelo T. Pallidum em qualquer fase da história natural da infecção, cuja mortalidade ultrapassa $60 \%$. Em pessoas com HIV, essa complicação pode ser mais frequente, surgir após um menor período de latência e ser mais agressiva. Na maioria das vezes, a neurossífilis é assintomática ou se apresenta por meio de manifestações clínicas pouco específicas que demandam alto índice de suspeição e propedêutica laboratorial invasiva. O diagnóstico é feito através de provas sorológicas ou de biologia molecular no líquor. A controvérsia é sobre a indicação desse diagnóstico e respectivo tratamento nos casos assintomáticos, principalmente em se tratando de pessoas que vivem com o HIV. Esta revisão narrativa da literatura concluiu que a punção lombar propedêutica da neurossífilis está indicada em pessoas que apresentam sororreagência ao VDRL, associado a uma ou mais das seguintes condições: manifestações neurológicas na esfera motora, sensitiva ou cognitiva; sintomatologia sifilítica ou VDRL maior que 1:8 pós-tratamento, descartada reinfecção; pessoas com HIV na presença de CD4 menor que 351 células $/ \mathrm{mm}^{3}$ e VDRL maior ou igual a 1:32; e evidência de doença sifilítica terciária não neurológica em atividade (goma ou aortite). Feito o diagnóstico a partir desses critérios, o tratamento específico deverá ser administrado mesmo nos casos assintomáticos.

Palavras-chave: sífilis; neurossífilis; HIV; líquido cefalorraquidiano; punção espinal.

\begin{abstract}
Syphilis is an infect-contagious injury caused by Treponema pallidum, of predominantly sexual transmission, which affects 12 million people worldwide each year. If not cured in its early stages, predisposes to potentially serious complications. One of the complications is neurosyphilis, characterized by the invasion of the central nervous system by $T$. pallidum in any phase of the natural history of the disease, whose mortality exceeds $60 \%$. In people with HIV, this complication may be more frequent, arising after a shorter period of latency and also be more aggressive. Most often, neurosyphilis is asymptomatic or presents itself by means of little specific clinical manifestations, which require high index of suspicion and invasive laboratory propaedeutics. The diagnosis is made by serological tests or molecular biology study on the cerebrospinal fluid. The controversy is about the indication of this diagnosis and respective treatment in asymptomatic cases, mainly in people living with HIV. This narrative review of the literature concluded that propaedeutic lumbar puncture of neurosyphilis is recommended for people who have serum-reactivity to the VDRL, associated with one or more of the following conditions: neurological manifestations on motor, sensory or cognitive sphere; syphilitic symptoms or VDRL greater than 1:8 post-treatment, discarded reinfection; persons with HIV at the presence of CD4 less than 351 cells $/ \mathrm{mm}^{3}$ and VDRL greater than or equal to $1: 32$; and evidence of no neurological tertiary syphilitic disease in activity (gumma or aortitis). Made the diagnose from these criteria, specific treatment should be given even in asymptomatic cases.
\end{abstract}

Keywords: syphilis; neurosyphilis; HIV; cerebrospinal fluid; spinal puncture. 


\section{INTRODUÇÃO}

A sífilis é um agravo multissistêmico infectocontagioso causado pelo Treponema pallidum, de transmissão predominantemente sexual, e de imunopatogenia e manifestações clínicas complexas; porém, de fácil diagnóstico e tratamento curativo em fases iniciais. Apesar disso, ainda afeta 12 milhões de pessoas por ano em todo o mundo'

Classicamente começa por uma fase primária caracterizada pelo aparecimento de úlcera genital única e indolor após um período de incubação de até três meses. Essa lesão desaparece espontaneamente dentro de quatro semanas sem deixar cicatrizes. Se não tratada, evolui para sífilis secundária dentro de um prazo que varia de 4 a 8 semanas após o surgimento da úlcera genital. Nesta fase, manifesta-se por micropoliadenopatia, exantema morbiliforme não pruriginoso, pápulas palmo-plantares, leucoplacas mucosas, alopécia e condiloma plano. Se não for curada mediante tratamento feito prioritariamente com penicilina, poderá complicar-se com manifestações graves principalmente na pele, nos ossos, no sistema nervoso ou no aparelho cardiovascular, as quais surgem após um período de latência clínica geralmente superior a 10 anos e caracterizam a fase terciária da sífilis ${ }^{2}$.

A afecção do sistema nervoso central, denominada neurossífilis, surge em até $40 \%$ das pessoas que não tratam a sífilis em suas fases primária ou secundária, cuja mortalidade ultrapassa $60 \%$ dos casos. Pode ser assintomática, ou sintomática a partir de suas formas clínicas meningovascular, meningítica, gomosa cerebral ou medular, epileptiforme, neuro-óptica atrófica, com lesão do sétimo par craniano, com paralisia geral ou na forma de tabes dorsalis ${ }^{1,2}$.

Não existem dados epidemiológicos muito confiáveis sobre a epidemiologia da neurossífilis devido a dificuldades relacionadas à identificação e registro desse agravo; porém, é senso observacional comum que, a partir da introdução do uso de penicilinas para o tratamento da sífilis até o advento da AIDS, essa afecção terciária tornou-se rara. No entanto, com o surgimento e com a rápida propagação da infecção pelo vírus da imunodeficiência humana (HIV) a partir da década de 1980, observou-se reincremento do número de casos de neurossífilis observados na prática clínica ${ }^{1,3-6}$.

Ainda que a neurossífilis se caracterize pela invasão do Sistema Nervoso Central pelo T. pallidum, a maioria dos casos é assintomática, independentemente da coinfecção com o HIV. Contudo, como a infecção por esse vírus prejudica a resposta imune da pessoa contra o T. pallidum, teme-se por maior possibilidade de desenvolvimento de transtornos clínicos relacionados à neurossífilis em pessoas infectadas pelo HIV, bem como pela possibilidade de início precoce de sintomas neurológicos nesses indivíduos ${ }^{1,3}$.

Apesar da neurossífilis ser classificada como afecção terciária na história natural da doença, é reconhecida a invasão do sistema nervoso central pelo T. pallidum desde fases logo a partir da infecção. Por esse motivo, a tendência tem sido considerar essa invasão, ainda que assintomática, como neurossífilis, independentemente da fase evolutiva clínica em que a pessoa se encontra. Ou seja, a neurossífilis pode ser detectada laboratorialmente em qualquer estágio da história natural da infecção pelo T. pallidum ${ }^{1,5-7}$.
Por outro lado, tendo em vista a inespecificidade das manifestações clínicas e a elevada prevalência de casos assintomáticos, o diagnóstico torna-se muito dependente da realização de reações no líquor por ensaios que compreendem o VDRL, o FTA-Abs ou a pesquisa do T. pallidum por técnicas de biologia molecular ${ }^{1}$.

Problema semelhante é visto no seguimento pós-tratamento da neurossífilis, uma vez que pode exigir punção lombar semestral por dois anos em caso de afecção meníngea. No entanto, admite-se o seguimento por VDRL sérico desde que o diagnóstico liquórico tenha sido feito por pleocitose e sororreatividade ao VDRL, uma vez que, nessas condições, o resultado desse exame no sangue corresponde ao resultado esperado no líquor de pessoas tratadas para neurossífilis ${ }^{8}$. Contudo, essa correspondência não acontece de modo confiável em pessoas com HIV. Nesse caso, a punção lombar torna-se praticamente indispensável também para o seguimento ${ }^{1}$.

A questão que surge na atualidade é sobre a necessidade ou não desse diagnóstico e respectivo tratamento nos casos assintomáticos, principalmente em se tratando de pessoas que vivem com o HIV. Um dos motivos dessa preocupação é que a coinfecção HIV-T. Pallidum é relativamente comum ao ponto de exigir um número grande de punções na rotina clínica se indicado sistematicamente a todas as pessoas assim co-infectadas. $\mathrm{O}$ outro motivo é que a punção lombar é um procedimento invasivo que tem implicações médicas, econômicas e operacionais significativas ${ }^{1,3,6}$.

Nesse sentido, o presente artigo teve como objetivo apontar as indicações específicas para a realização de punção lombar para o diagnóstico de neurossífilis, segundo dados consolidados a partir de uma revisão narrativa de literatura.

\section{MÉTODOS}

Revisão narrativa da literatura a partir do descritor "neurossífilis" aplicado às seguintes bases de dados constantes na Bireme: LILACS, IBECS, MEDLINE, Biblioteca COCHRANE e SciELO. Foram encontrados 1.925 artigos publicados de 1922 a 2014, sem restrição de idioma. Desses, 432 eram textos completos; 279 deles publicados a partir de 2005. Entre esses de publicação mais recente, 16 foram referenciados neste artigo por tratarem de modo direto e específico do interesse desta revisão. Entre os 16, foram sete revisões narrativas da literatura, três ensaios clínicos, dois relatos de casos, dois estudos transversais retrospectivos, um estudo de coorte e uma revisão sistemática. Além desses artigos, também foram referenciados mais seis textos de apoio: três diretrizes de cuidado, dois livros e uma dissertação de mestrado.

\section{RESULTADO E DISCUSSÃO}

Uma pessoa com neurossífilis pode ser assintomática durante anos. A doença se manifesta mais frequentemente nas formas meníngea, vascular e parenquimatosa. Formas meníngeas e vasculares frequentemente ocorrem em conjunto, como na neurossífilis meningovascular que se manifesta como doença inflamatória. 
Já nas formas parenquimatosas os sintomas são primordialmente neurodegenerativos e incluem a tabes dorsalis e a paresia geral ${ }^{9}$.

Quanto à compreensão dos achados laboratoriais, é importante considerar a sensibilidade e a especificidade das provas imunológicas. A sensibilidade reflete o quanto o teste é eficaz em identificar corretamente os indivíduos realmente acometidos pelo agravo, podendo ser tão alta ao ponto de apresentar resultados falso-positivos ${ }^{10}$.

Portanto, um teste de alta sensibilidade é essencial para não descartar um caso verdadeiro. Já a especificidade reflete o quanto a prova é eficaz em identificar corretamente indivíduos que não apresentam a condição de interesse. Ou seja, o quanto o teste é capaz de refutar o diagnóstico entre os que não apresentam a condição. O ideal é que os testes tenham elevada sensibilidade e especificidade ao mesmo tempo; isto é, elevada acurácia em confirmar o diagnóstico em doentes e descartá-lo nos não doentes ${ }^{10}$.

No caso da neurossífilis, a pesquisa por meio de reações treponêmicas (FTA-Abs) e não treponêmicas (VDRL) realizadas simultaneamente no líquido cefalorraquidiano (LCR) é a estratégia mais acurada para o diagnóstico. O motivo é que o VDRL tem elevada especificidade e o FTA-Abs alta sensibilidade. Assim, VDRL positivo no líquor confirma o diagnóstico de neurossífilis enquanto que negativo não descarta, ao passo que o FTA-Abs positivo nesse espécime não confirma o diagnóstico, mas, descarta-o em caso de resultado não reagente. Um cuidado que se recomenda é que, em casos de acidentes de punção com contaminação sanguínea do líquor, o VDRL poderá tornar-se falso-positivo nesse material devido à sororreagência e não liquorreagência ${ }^{11}$.

Uma vez realizado o diagnóstico, é necessário o tratamento imediato com Penicilina Cristalina, 3-4 milhões U IV 4/4 horas por 14 dias. Para os alérgicos à penicilina, pode-se usar Ceftriaxone $2 \mathrm{~g} /$ dia por 14 dias ou Doxiciclina $100 \mathrm{mg}$ de 12/12 h por 28 dias, ou realizar a dessensibilização à penicilina ${ }^{3,8,12,13}$.

Postas essas considerações, o exame do LCR deverá ser indicado nas pessoas com diagnóstico sorológico de sífilis e sintomas neurológicos, e naquelas que mantêm VDRL sérico reagente em títulos maiores ou iguais a 1:8 ou sintomas persistentes ou recorrentes após o tratamento correto. Nas pessoas coinfectadas com o HIV, além desses critérios, CD4 menor que 351 células $/ \mathrm{mm}^{3}$ e VDRL maior que 1:32 também é indicação para punção lombar diagnóstica ${ }^{8}$.

A esse respeito, os manuais de controle da sífilis indicam diagnóstico liquórico em indivíduos assintomáticos com sífilis tardia latente (assintomáticos com mais de um ano de início da doença ou com duração desconhecida), e nos que vivem com o HIV independentemente do estágio ou sintomas da doença. Contudo, essa indicação encontra controvérsias na literatura ${ }^{14,15}$.

Em síntese, nenhum teste imunológico no líquor isoladamente é seguro para o diagnóstico da neurossífilis, o qual depende da combinação de positividade ao VDRL e ao FTA-Abs, aumento da celularidade liquórica com pleocitose (maior que 10 linfócitos/ $\mathrm{mL}$ líquor) e elevação proteica no LCR superior a $40 \mathrm{mg} / \mathrm{dL}^{14,16}$.

Vale lembrar que em pessoas que vivem com o HIV, alterações proteicas e celulares podem ocorrer no LCR devido ao próprio
HIV. Além disso, esses sujeitos têm maior chance de desenvolver neurossífilis clínica a partir de uma condição assintomática ${ }^{17}$.

Portanto, é muito importante afastar o diagnóstico de invasão do Sistema Nervoso Central (SNC), especialmente, em quem vive com o HIV, por meio da combinação da especificidade do VDRL com a sensibilidade do FTA-Abs ${ }^{14,18}$. A pesquisa de T. pallidum no líquor por meio de reação em cadeia da polimerase é uma opção a ser considerada em pessoas que vivem com o HIV, uma vez que a acurácia do VDRL e do FTA-Abs nesses indivíduos é reduzida comparativamente a quem não vive com o HIV ${ }^{1}$.

Outra consideração importante é a possibilidade do efeito Prozona que pode ocorrer em de 1 a $2 \%$ dos sujeitos. Esse efeito é um falso-negativo que pode ser visto com o VDRL devido a uma concentração de anticorpo no espécime testado muito alta em relação à concentração de antígeno no aglutinante do teste. Esse excesso desproporcional de anticorpos pode inibir a aglutinação indicadora da positividade da reação. Nesse caso, aconselham-se testagens sequenciais em amostras progressivamente diluídas na tentativa de reduzir essa diferença na concentração de anticorpos no espécime em relação à de antígenos no reagente, em busca de uma positividade no teste em diluições mais altas ${ }^{14}$

No que diz respeito ao acompanhamento pós-tratamento nas pessoas que vivem com o HIV, espera-se que o VDRL liquórico normalize 2,5 vezes menos do que naquelas que não têm esse vírus. Nessas pessoas, quando a concentração sérica de CD4 é inferior a 200 células $/ \mathrm{mm}^{3}$, o VDRL regulariza 3,7 vezes menos que naquelas que têm mais de 200 dessas células $/ \mathrm{mm}^{6,18}$.

Frente ao exposto, a literatura procura racionalizar a indicação de punção lombar diagnóstica da neurossífilis, propondo esse procedimento para pessoas que apresentam sororreagência ao VDRL $^{8,12,15-21}$ (Quadro 1).

\section{CONCLUSÃO}

Por se tratar de uma revisão narrativa da literatura, este artigo não é uma referência de evidência científica sobre o tema discutido. Como um consolidado de publicações obtidas a partir de um conjunto de dados bibliográficos, organizado e apresentado segundo critérios próprios dos autores, expõe, de modo sistematizado, uma parte dos argumentos sobre a aplicabilidade do diagnóstico liquórico da neurossífilis e suas indicações de maior consenso.

Quadro 1: Procedimento para pessoas que apresentam sororreagência ao VDRL

Sintomas neurológicos na esfera motora, sensitiva ou cognitiva. Persistência ou recorrência dos sintomas da sífilis após tratamento, independente da fase clínica.

Manutenção de títulos altos de VDRL>1:8 no sexto mês de seguimento, caso não haja reinfecção.

$V D R L \geq 1: 8$ na última dosagem do seguimento de cada fase clínica. Pessoas que vivem com o HIV e apresentem VDRL reator em diluições a partir de 1:32 e estejam com CD4 $\leq 350$.

Evidência de doença sifilítica terciária não neurológica em atividade (goma ou aortite). 
Assim, encontrou-se que tal diagnóstico pode ser feito com razoável certeza quando houver síndrome neuropsiquiátrica associada com VDRL liquórico positivo. Se o VDRL for negativo no líquor, a liquorreagência ao FTA-Abs associada ao aumento da celularidade, das proteínas ou do IgG nesse espécime permite a confirmação diagnóstica da neurossífilis ${ }^{18}$.

Especificamente em relação a quem vive com o HIV e tem sororreagência ao VDRL, a punção liquórica em todos esses sujeitos é controversa apesar de recomendada nos manuais de atenção a essas pessoas. As diretrizes especificam indicações para esse procedimento que, entretanto, são pouco claras quanto à recomendação em certas situações clínicas como sífilis precoce sem envolvimento neurológico, ou em situações de oscilação rápida do CD4.
Por outro lado, testes treponêmicos não reativos no LCR excluem o diagnóstico de envolvimento do SNC pela sífilis e, talvez, devam ser valorizados em caso de pessoas assintomáticas que tenham risco elevado para o desenvolvimento de neurossífilis, ou de pessoas sob suspeita significativamente duvidosa $a^{5,9,15,18,19}$.

Reforça-se que pessoas que vivem com o HIV têm maior probabilidade de desenvolver neurossífilis clínica, o que torna importante certificar-se da invasão ou não do SNC, especialmente em quem têm CD4 menor que 351 células/ $\mathrm{mm}^{3}$ e sororreagência ao VDRL ${ }^{4,6,20,22}$. Ainda assim, é de bom alvitre a adoção de critérios específicos para a indicação de punção lombar diagnóstica nesses sujeitos, de sorte a evitar-se esse procedimento em situações em que a probabilidade de complicação neurossifilítica seja menor.

\section{REFERÊNCIAS}

1. Fraga DD. Detecção de Treponema pallidum em líquido cefalorraquidinao (LCR) por reação em cadeia da polimerase (PCR) em pacientes HIV positivos assintomáticos com diagnóstico de sífilis latente. Dissertação (Mestrado) - Universidade Federal do Rio Grande do Sul. Porto Alegre: 2013.

2. Brasil. Ministério da Saúde. Secretaria de Vigilância em Saúde. Departamento de Vigilância Epidemiológica. Doenças infecciosas e parasitárias: guia de bolso. Ministério da Saúde. 8 ed. rev. Brasília: Ministério da Saúde, 2010. p. 373-86.

3. Silva Neto RP. Erros diagnóstico e terapêutico em neurossífilis: uma análise de dez anos. Rev Bras Neurol. 2008;44(2):35-40.

4. Ghanem KG. Review: neurosyphilis: a historical perspective and review. CNS Neurosc Ther. 2010;16(5):e157-68. http://dx.doi.org/10.1111/j.1755-5949.2010.00183.x

5. Madhusudhan M. Neurosyphilis. Neurol India; J Neurol Soc India. 2009;57(3):233-4.

http://dx.doi.org/10.4103/0028-3886.53259

6. De Almeida SM, Bhatt A, Riggs PK, Durelle J, Lazzaretto D, Marquie-Beck J, et al. Cerebrospinal fluid human immunodeficiency vírus viral load in patients with neurosyphilis. J Neurovirol. 2010;16(1):6-12

http://dx.doi.org/10.3109/13550280903514776

7. Proudfoot M, McLean B. Old adversaries, modern mistakes: neurosyphilis. Pract Neurol. 2013;13(3):174-7. http://dx.doi.org/10.1136/practneurol-2012-000314

8. Cleinman IB, May SB. Diretrizes de Atendimento de Sífilis em Adultos. Serviço de Doenças Infecciosas e Parasitárias do Hospital Universitário Clementino Fraga Filho. Universidade Federal do Rio de Janeiro. Disponível em: http://www.hucff.ufrj.br/ download-de-arquivos/category/26-dip?download=338: rotinas. Acesso em: 13 mar. 2015.

9. Chahine LM, Khoriaty RN, Tomford WJ, Hussain MS. The changing face of neurosyphilis. Int J Stroke. 2011;6(2):136-43. http://dx.doi.org/10.1111/j.1747-4949.2010.00568.x

10. Almeida Filho N, Rouquayrol, MZ. Introdução à Epidemiologia. 4. ed. Rio de Janeiro: MESI; 2006

11. Barros AM, Cunha AP, Lisboa C, Sá MJ, Resende C. Neurossífilis revisão clínica e laboratorial. Arq Med. 2005;19(3):121-9.
12. WorkowskiKA, Berman SM. Sexuallytransmitted diseases treatment guidelines, 2006. MMWR Recomm Rep. 2006;55(RR11):1-94.

13. Workowski KA, Berman S. Sexually transmitted diseases treatment guidelines, 2010. MMWR Recomm Rep. 2010;59(RR12):1-110.

14. Avelleira JCR, Bottino G. Sífilis: diagnóstico, tratamento e controle. An Bras Dermatol. 2006;81(2):111-26. http://dx.doi.org/10.1590/S0365-05962006000200002

15. Machado LR, Livramento JA, Vianna LS. O exame do liquido cefalorraquidiano em doenças infecciosas do sistema nervoso: quando pedir, o que pedir, o que esperar. Arq Neuropsiquiatr. 2013;71(9B):693-98.

http://dx.doi.org/10.1590/0004-282X20130153

16. Ho EL, Marra CM. Treponemal tests for neurosyphilis - less accurate than what we thought? Sex Transm Dis. 2012;39(4):298-9. http://dx.doi.org/10.1097/OLQ.0b013e31824ee574

17. Salazar JC, Cruz AR, Pope CD, Valderrama L, Trujillo R, Saravia $N G$, et al. Treponema pallidum elicits innate and adaptive cellular immuneresponses in skin and blood during secondary syphilis: a flow-cytometric analysis. J Infect Dis. 2007;195(6): 879-87. http://dx.doi.org/10.1086/511822

18. Nadal LRM, Nadal SR. Indicações da punção liquórica nos portadores de sífilis. Rev Bras Colo-proctol. 2006;26(4):459-62. http://dx.doi.org/10.1590/S0101-98802006000400015

19. Ghanem KG, Moore RD, Rompalo AM, Erbelding EJ, Zenilman JM, Gebo KA. Lumbar puncture in HIV-infected patients with syphilis and no neurologic symptoms. Clin Infect Dis. 2009;48(6):816-21. http://dx.doi.org/10.1086/597096

20. Dumaresq J, Langevin S, Gagnon S, Serhir B, Deligne B, Tremblay C, et al. Clinical prediction and diagnosis of neurosyphilis in HIV-infected patients with early Syphilis. J Clin Microbiol. 2013;51(12):4060-6. http://dx.doi.org/10.1128/JCM.01989-13

21. Martinelli P, Rizzo G, Scaglione C, Capellari S. Neurosyphilis orofacial dyskinesia: the candy sign. Mov Disord. 2013;28(2):246-7. http://dx.doi.org/10.1002/mds.25281

22. Choe PG, Song JS, Song KH, Jeon JH, Park WB, Park KU, et al. Usefulness of routine lumbar puncture in non-HIV patients with latent syphilis of unknown duration. Sex Transm Infect. 2010;86(1):39-40. http://dx.doi.org/10.1136/sti.2009.037267 